\title{
Bradycardia and asystole during intubation in a child with Dandy-Walker syndrome for an emergency operation at night : The possible causes
}

Asako Nagane, Satoshi Naruse, Masataka Kuroki, Toshinari Suzuki Department of Anesthesiology, Saitama Medical Center, Saitama Medical University, JAPAN

\section{Backgrounds}

Dandy-Walker syndrome (DWS) is a congenital disease characterized by absence of cerebellar vermis and cyst development in the posterior fossa communicating with the fourth ventricle, resulting in hydrocephalus. Anesthesia for DWS child may be challenging due to difficult airway. We report a case of bradycardia and asystole occurring at intubation in a child with DWS undergoing emergency shunt revision at night, in which cause of cardiac arrest was difficult to determine.

\section{Case Report}

1 year-old female, body weight $5 \mathrm{~kg}$, with DWS diagnosed at birth. Cyst-peritoneal shunt was placed at 4 months old. This time, she was undergoing emergency cyst drainage because of shunt obstruction, enlargement of a cyst in the posterior fossa (fig.1,2) and increased posterior fossa intracranial pressure (ICP). She had episodes of bradycardia while being taken care of in ward in the supine position, and osmotic diuretic (20\% mannitol) was given div. for reduction of ICF Intraoperative course (fig.3): She was placed in the supine position with the head turned to the left on OR table for induction of anesthesia.

23:14 Induction of anesthesia (fentanyl $10 \mu \mathrm{g} /$ midazolam $0.5 \mathrm{mg} /$ rocuronium $3 \mathrm{mg}$ ), sevoflurane $2.5 \%$

23:20 Laryngoscopy was performed with difficulty; CormackLehane grade IIb.

She was then placed in the supine position facing midline. Sudden bradycardia during laryngoscopy,

intubated the trachea with $4.0 \mathrm{~mm}$ cuffed endotracheal tube, atropine $0.1 \mathrm{mg}^{*} 3 \mathrm{iv}$.

23:21 Desaturation, cardiac arrest $\rightarrow$ chest compression

23:22 Epinephrine $0.125 \mathrm{mg}$ iv.

Chest wall was raised, but $\mathrm{ETCO}_{2}$ was not detected.

23:33 Reintubation with $3.5 \mathrm{~mm}$ uncuffed ETT.

23:34 Epinephrine 0.125mg iv., $\mathrm{ETCO}_{2}$ still not detected.

23:43 Reintubation with $3.0 \mathrm{~mm}$ cuffed ETT. Placement of the

ET tube was finally confirmed fiberoptically.

She was positioned in a lateral position to decrease ICP.

23:44 Return of spontaneous circulation

Drainage operation through a burr hole for the Dandy-Walker cyst proceeded uneventfully thereafter.

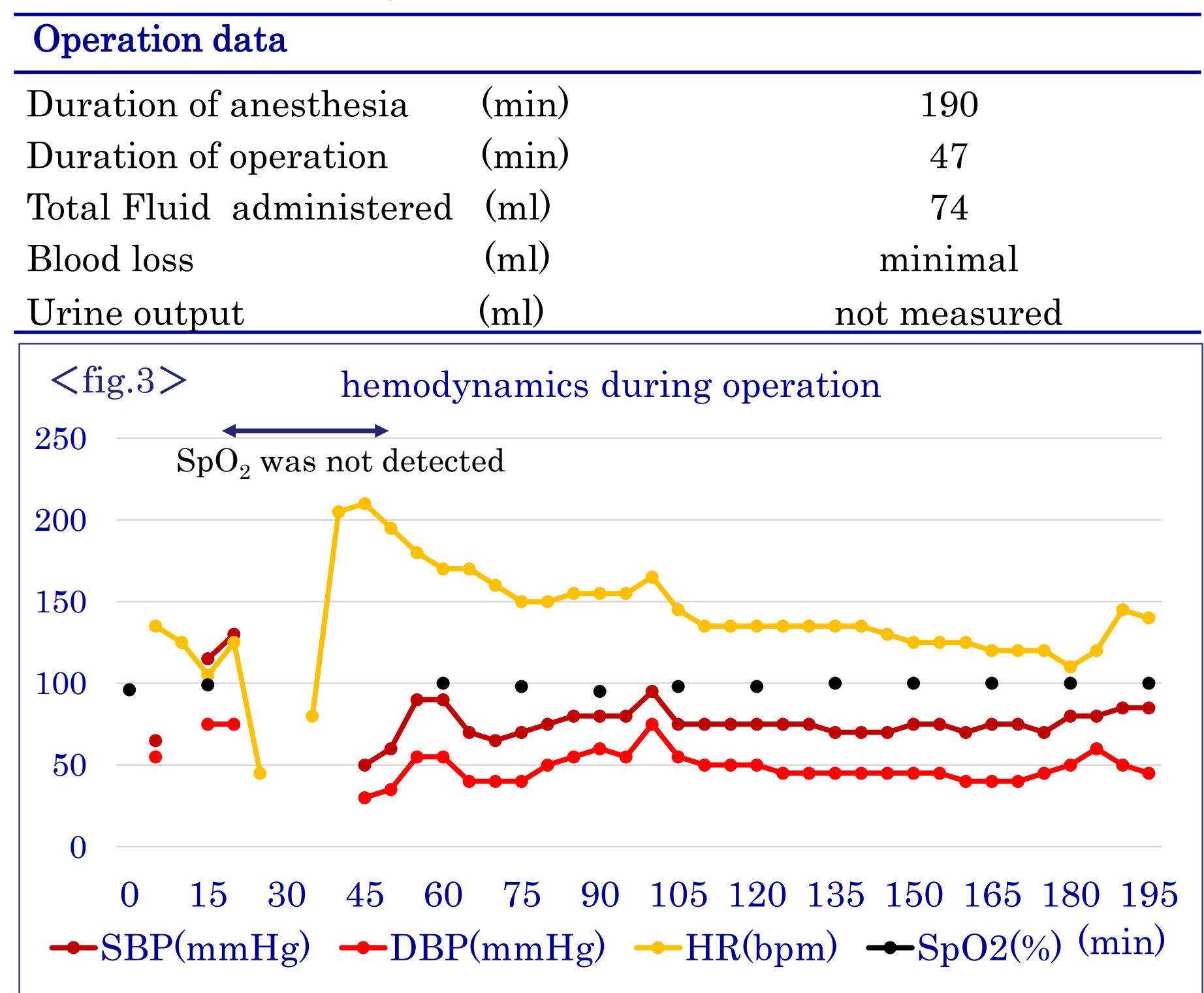

\section{Postoperative Course / Outcome}

Day 6

Day 15

Right-sided cyst-peritoneal (CP) shunt was placed

Pt's trachea was extubated.

spontaneous eye opening, moves all limbs, spontaneous

breathing, stable vital signs

Day 43 Drainage tube exchange

Day 57 Left-sided CP shunt was placed

Day 83 no postoperative complications, weaning from the ventilator and transfer to outside hospital

Readmission due to bronchitis, tube feeding, transfer to outside hospital,

Currently, the patient is 2 years and 10 months old.

\section{Discussion}

$<$ Diagnosis and clinical signs of Dandy-Walker syndrome > The most common revealing signs of DWS are the characteristic enlargement of the occiput, macrocrania, mental retardation, cerebellar ataxia, and those of increased ICP. Cerebral anomalies may lead to respiratory failure, such as apneic spells. About one-fourth of such patients exhibit these signs in the neonatal period. Associated congenital anomalies are craniofacial, cardiac, renal, and skeletal abnormalities. Diagnosis of Dandy-Walker syndrome is usually easy with clinical signs, CT, or MRI.

< Problem of maintenance of anesthesia for DWS >

Intubation should be performed under general anesthesia to minimize effect on ICP during intubation. However, tracheal intubation or airway management is often difficult due to enlarged head size and craniofacial malformations such as micrognathia or cleft palate. Awake intubation is required despite the increased ICP if difficulties in airway management are anticipated.

<Anesthetic course in this patient >

Anesthetic planning and experience of anesthesiologists were inadequate due to the emergency nature of the operation at night.

She was placed in a lateral position for induction of anesthesia because of increased ICP. However, difficult laryngoscopy necessitated supine positioning for intubation.

The likely causes of bradycardia and cardiac arrest in this child may be questionable intubation as well as sudden increased ICP (supine position) via Cushing's reflex.

However, cardiac arrest due to elevated ICP in neurosurgical patients is reportedly rare ${ }^{1)}$.

Thus, compressing the brainstem through suture disjunction may also lead to severe hemodynamic disturbances.

This case was successfully treated with immediate CPR, repositioning to a lateral position, and opening of the dura to decrease ICP.

$<$ fig. $1>$

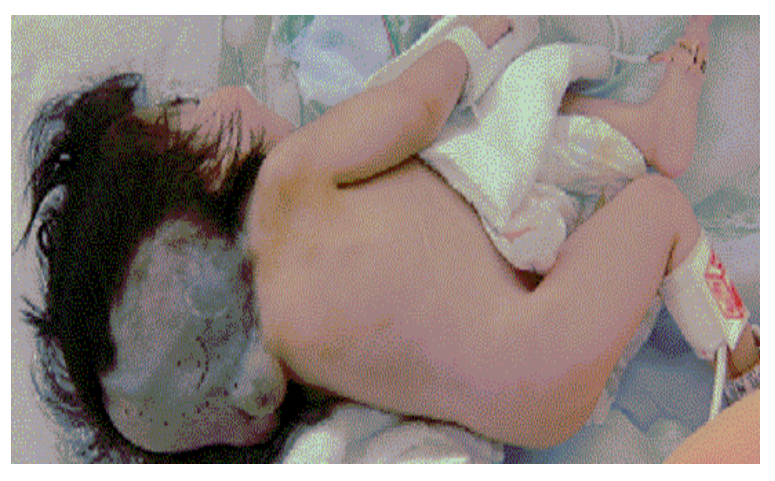
$<$ fig. $2(\mathrm{CT})>$

Disjunction of the skull suture

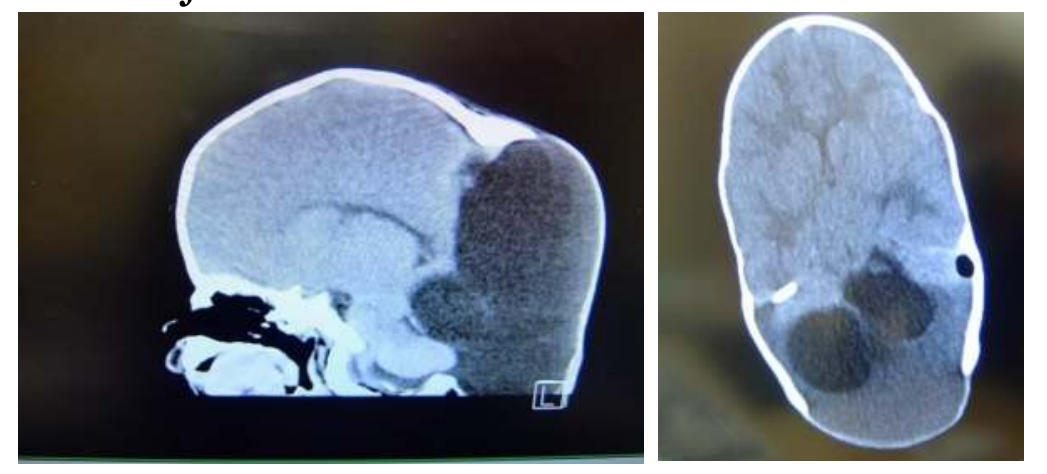

\section{Learning points}

- We report the anesthetic management of a child with DWS for an emergency operation at night.

- This case highlights the importance of thorough preoperative evaluation, atropine premedication, and adjusting the body position, even for an emergency operation at night, in order to prevent ICP increase, compression of the brainstem through suture disjunction, asystole, bradycardia, and difficult airway. 